\title{
Traceability of Voltage Measurements for Non-Sinusoidal Waveforms.
}

\author{
P. Espel, A. Poletaeff, H. Ndilimabaka \\ Laboratoire national de métrologie et d’essais, 29 avenue Roger Hennequin, 78197 Trappes, France, patrick.espel@lne.fr \\ This paper describes the result of work performed at the Laboratoire National de Métrologie et d'Essais (LNE) aiming at \\ developing a standard system to measure RMS value and harmonic contents of distorted voltage waveforms by means of a \\ sampling voltmeter. Thermal converters are used to trace the RMS value to the SI units. The error of the DVM has been generally \\ found less than $10 \mu \mathrm{V} / \mathrm{V}$ up to $2 \mathrm{kHz}$ but can reach about $50 \mu \mathrm{V} / \mathrm{V}$ at $2.5 \mathrm{kHz}$ for RMS voltage measurements for sine waves. For \\ distorted waveforms, deviations within $15 \mu \mathrm{V} / \mathrm{V}$ have been obtained whatever the total harmonic distortion of the waveforms.
}

Keywords: distorted voltage waveforms, thermal converters, sampling techniques, analog to digital converters.

\section{INTRODUCTION}

$\mathrm{T}$ HE MEASUREMENTS of root mean square (RMS) of sinusoidal signals with uncertainties of a few $\mu \mathrm{V} / \mathrm{V}$ are carried out in most National Metrology Institutes (NMIs) by using thermal converters. Indeed, these thermal converters are widely used to trace AC quantities (AC currents and voltages) to the corresponding DC quantities with the best level of uncertainty [1]. Agreement of some parts in $10^{7}$ to 15 part in $10^{6}$, in the frequency range from 1 $\mathrm{kHz}$ to $1 \mathrm{MHz}$, has been found between various NMIs at voltages ranging from $1 \mathrm{~V}$ to $3 \mathrm{~V}$ [2]. Below $1 \mathrm{kHz}$ frequency, uncertainties of 1 or 2 parts in $10^{6}$ are currently obtained and are higher down to $10 \mathrm{~Hz}$. Although they are the most accurate true RMS measuring devices available, they suffer from many drawbacks: their low impedance, their low dynamic range and their long measuring times. Moreover, they do not give information about the harmonic contents of the signals such as amplitude and phase of each harmonic, total harmonic distortion...

All these quantities are really very important since over the past few years new requirements for electricity supply quality including low harmonic distortion have appeared. As a consequence power analyzers are increasingly being used to insure the quality of the grids. This has created a need for national laboratories to provide calibration facilities and traceability for the harmonic related quantities measured by these new instruments.

For all these reasons, one alternative to thermal converters is the use of sampling methods and analog-to-digital converters (ADCs) of high accuracy with small integration times, especially those from the HP 3458 A* sampling digital voltmeter (DVM), which is commonly used in most NMIs. The objective of the study is to measure the total RMS value $U_{\text {eff }}$ of distorted waveforms by means of a sampling voltmeter as well as the RMS value of each harmonic and the total harmonic distortion. Thermal converters are only used to trace the quantity $U_{\text {eff }}$ to the SI units.

\section{DEFINITIONS}

The distorted voltage waveforms have a dominant fundamental component at $50 \mathrm{~Hz}$ and the harmonic content is limited to the first 50 stationary harmonics (periodic signals). These waveforms can be decomposed into Fourier series as:

$$
u(t)=\sum_{k=1}^{\infty} U_{k} \sin \left(2 \pi f_{k} t+\varphi_{k}\right)
$$

$U_{1}, U_{k}$ are the amplitudes of the fundamental and the $k^{\text {th }}$ harmonic component which frequencies are respectively $f_{1}$ and $f_{k}=k f_{1}$.

The two quantities of interest for our work are :

- the RMS value $U_{\text {eff }}$ of the voltage signal which is defined as:

$$
U_{e f f}=\left(U_{1}^{2}+U_{2}^{2}+\ldots+U_{k}^{2}+\ldots\right)^{\frac{1}{2}}
$$

- the total harmonic distortion of the signal, often abbreviated as THD which can be calculated in one of two different ways:

$T H D=\left(\frac{\sum_{k>1}^{\infty} U_{k}^{2}}{U_{e f f}^{2}}\right)^{\frac{1}{2}}$ or $T H D=\left(\frac{\sum_{k>1}^{\infty} U_{k}^{2}}{U_{1}^{2}}\right)^{\frac{1}{2}}$

The first definition is based on comparing the rms value of the harmonics to the total rms value while the second definition compares the rms value of the harmonics to the rms value of the fundamental component. In most situations, the difference will be negligible, but for highly distorted signals, it may be of importance.

In this paper, we have adopted the second definition which is the most commonly used. 


\section{MEASUREMENT METHOD}

\section{A. Basic measuring principle of the AC-DC thermal transfer}

The principle of AC-DC thermal transfer measurements is based on the comparison of the heating of a resistor produced by the successive application of AC and DC signals and measured by the means of a thermocouple fixed on the resistor. The procedure is the following : the AC signal to be measured is first applied to the heater resistor and the output voltage of the thermocouple is measured. Then the AC signal is replaced by a DC signal which is adjusted to produce the same output voltage of the thermocouple as previously. When this condition is fulfilled the DC signal is measured and it can be concluded that the RMS value of the AC signal is equal to the measured value of the DC signal.

Nevertheless since the response of a thermal converter is different for AC and DC signals, a correction called the ACDC transfer difference has to be applied. The AC-DC transfer difference $\delta$ of a thermal converter is defined by :

$$
\delta=\frac{E_{a c}-E_{d c}}{E_{d c}}
$$

where $E_{a c}$ is the RMS value of the AC signal applied to the thermal converter and $E_{d c}$ is the value of the DC signal which produces the same output voltage of the thermocouple. In practice, DC signal is applied successively in both polarities to eliminate the reversal error arising from asymmetry in the construction of the thermal converter. $E_{c}$ then represents the mean value of $E_{c}+$ and $E_{c^{-}}$.

The AC-DC transfer difference $\delta$ of the thermal converter used for the experiment is plotted in Fig. 1 as a function of the frequency $f$ of the signal measured. The uncertainties attainable by this technique are of the order of $1 \mu \mathrm{V} / \mathrm{V}$ for measurements at voltages of about $1 \mathrm{~V}$ at low frequency.

\section{B. Experimental setup}

The block diagram of the set-up is given in Fig.2. The sampling voltmeter (DVM) used for the measurement of the RMS value $U_{\text {eff }}$ and the reference thermal converter are connected in parallel and can be feeded by an AC or a DC source by means of an AC-DC switch. The output voltage of the thermal converter is measured by a precision nanovoltmeter. The sampling voltmeter is periodically calibrated against a Zener source as it also serves to measure the voltage delivered by the DC source (reference voltage).

The sequence AC, DC+, DC- and AC is then applied. Voltages measured by both the DVM and the precision nano-voltmeter (output voltage of the thermal converter) are each time recorded. The error of the DVM is computed from this set of data.

The AC source used to generate the distorted waveforms is a FLUKE 6100A* Electrical Power Standard Master unit which also provides a "sample reference" (TTL) at its rear panel to trigger the sampling DVM (Fig.3). The TTL reference signal is a harmonic of the phase reference signal and is phase locked to it. Thus the frequency of the power source and the sampling rate are synchronized, in order to take an integer number of periods.

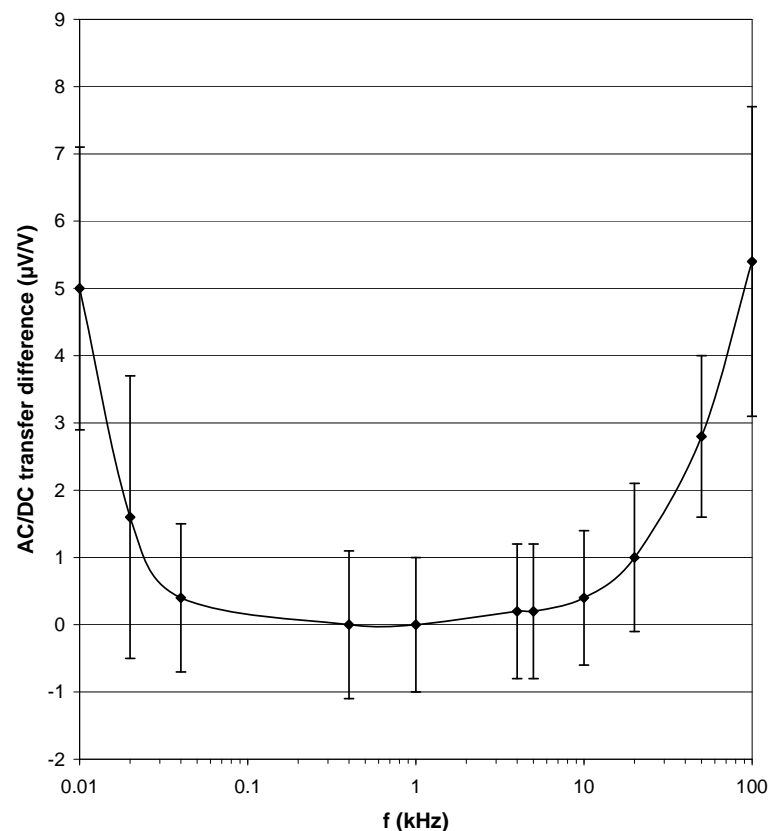

Fig.1. AC-DC transfer difference $\delta$ of the thermal converter

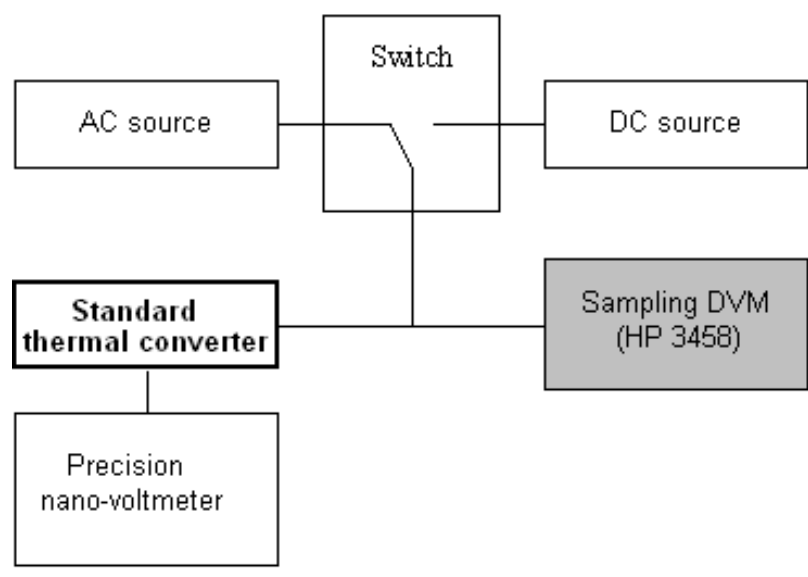

Fig.2. Experimental set-up.

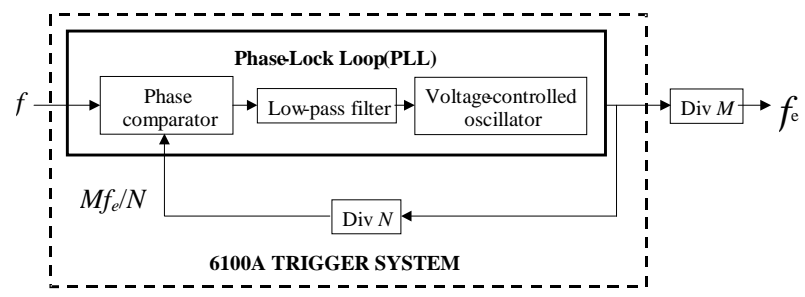

Fig.3. Trigger control with phase-locking.

Using this "sample reference", it is not possible to select any number $N$ of samples and any number $M$ of periods. For example, at $53 \mathrm{~Hz}$, we get by default exactly 1024 samples over 1 period or 2048 samples over 2 periods and so on. The sampling frequency is $54.3 \mathrm{kHz}$ and can not be modified. Therefore, the analog-to-digital converter's integration time (or aperture time $T_{a}$ ) which corresponds to the integration of the signal over a finite time is limited. So, some frequency 
dividers have been inserted in the experimental set-up in order to divide the sampling frequency for getting exactly 1024 samples over 3, 5 or 7 periods (Fig.2) which gives sampling rates respectively at about $18 \mathrm{kHz}, 10.8 \mathrm{kHz}$ and $7.7 \mathrm{kHz}$. This allows to increase the aperture time and therefore amplitude resolution.

For AC voltage measurements, the DVM is used in the DCV sampling mode. $N$ samples are taken at equally spaced discrete instants, covering an integer odd number $M$ of periods of the input waveform. The sampling frequency $F_{e}$ is $10.8 \mathrm{kHz}$. It is assumed that, at half the sampling frequency and above, the amplitudes of all spectral components are smaller than the resolution of the sampler. This is the mandatory condition for aliasing to be avoided. Then, the DVM records all the samples in reading memory using DINT format (32 bit integer format) and transfers the data to the controller using the DINT output format. The maximum number of samples is limited by the $20 \mathrm{kBytes}$ storage capacity of the multimeters. During the sampling, the autozero function and the display of the multimeters are disabled.



Fig.4. Error $e_{B P}$ due to limited bandwidth according to the frequency $f_{k}$ of the harmonics.

The samples are then analyzed by applying discrete Fourier transform (DFT) and all the components of the signal are deduced from the amplitude spectrum. Using the DVM in the DCV sampling mode, the main amplitude errors are due to the limited bandwidth and the non zero aperture time $T_{a}$ :

- because of the limited bandwidth of the DVM, the behavior of its input channel is similar to a second order low pass filter composed of two $5 \mathrm{k} \Omega$ resistances and two $82 \mathrm{pF}$ capacitances [3]. This filter changes the magnitude of each sample and the resulting error has been corrected by applying a factor $e_{B P}$ given by:

$$
e_{B P}=\frac{1}{\sqrt{1+7\left(2 \pi f_{k} R C\right)^{2}+\left(2 \pi f_{k} R C\right)^{4}}}
$$

Fig.4 shows this error according to the frequency $f_{k}$ of the harmonics. The harmonic content being limited to 50 stationary harmonics, the higher frequency considered is $2500 \mathrm{~Hz}$ and the resulting error is $145 \mu \mathrm{V} / \mathrm{V}$.

- the other error source is due to the non-zero aperture time $T_{a}$. The mean value of the signal integrated over this finite time does not correspond exactly to its value at the centre of the integration interval.

The DFT of a sampled signal may be defined by:

$$
\begin{gathered}
X(k f)=\sum_{n=0}^{N-1} x\left(n T_{e}\right) \exp \left(-2 \pi j \frac{k n}{N}\right) \\
k=0,1,2, \ldots ., N-1 .
\end{gathered}
$$

where $N$ is the number of samples, $T_{e}$ is the sampling period, $F_{e}$ is the sampling rate, $x\left(n T_{e}\right)$ is the input signal amplitude at time $n T_{e}$ and $X(\mathrm{kf})$ is the spectrum of $x$ at frequency $\mathrm{kf}$.

According to Poisson's formula, equation (6) can also be written as :

$$
X(k f)=X(f) * F_{e} \cdot \sum_{k=0}^{N-1} \delta\left(f-k F_{e}\right)
$$

where $\delta$ is the Dirac function.

Taking into account the aperture time $T_{a}$, the input signal amplitude $x\left(n T_{e}\right)$ can be expressed as :

$$
x\left(n T_{e}\right)=\frac{1}{T_{a}} \int_{n T_{e}-\frac{T_{a}}{2}}^{n T_{e}+\frac{T_{a}}{2}} x(t) d t
$$

and equation (7) becomes :

$$
X(k f)=\left[X(f) \frac{\sin \left(\pi f T_{a}\right)}{\pi f T_{a}} \exp \left(-2 \pi j v \frac{T_{a}}{2}\right)\right] * F_{e} \cdot \sum_{n=-\infty}^{+\infty} \delta\left(f-n F_{e}\right)
$$

Then, the resulting amplitude error is corrected by applying the factor $e_{T a}$ defined as:

$$
e_{T_{a}}=\frac{\sin \left(\pi f_{k} T_{a}\right)}{\pi f_{k} T_{a}}
$$

As shown in Fig.5, the error due to non-zero aperture time can be very large. For example, considering a signal frequency of $2500 \mathrm{~Hz}$ and an aperture time of $60 \mu \mathrm{s}$, this error is $36602 \mu \mathrm{V} / \mathrm{V}$.

These two systematic errors have been taken into account in the determination of the rms value of all harmonic components. The rms value of the distorted signal has been calculated as the quadratic sum of all harmonics. 


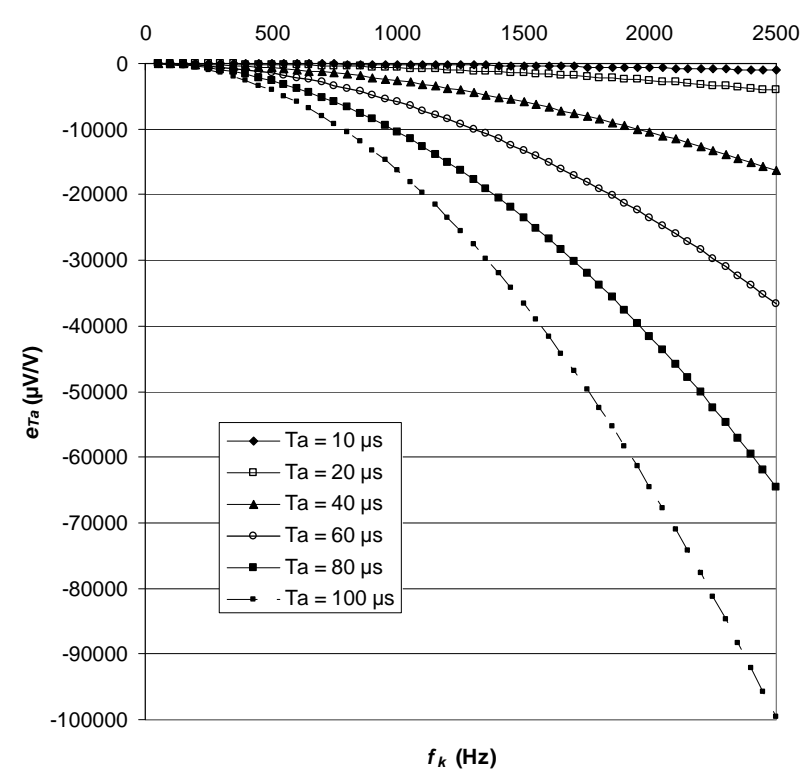

Fig.5. Error $e_{T a}$ due to aperture time according to the frequency $f_{k}$ of the harmonics.

\section{EXPERIMENTAL RESULTS}

A. Characterization of the analog-to-digital converters (ADC) of the DVM

The ADCs of the DVM have already been characterized for static [4] and dynamic [5] conditions, for sine waves at frequencies up to $400 \mathrm{~Hz}$. In this frequency range, selecting optimized sampling parameters, the agreement between the sampling technique and AC-DC transfer using thermal converters has been found better than $3 \mu \mathrm{V} / \mathrm{V}$.

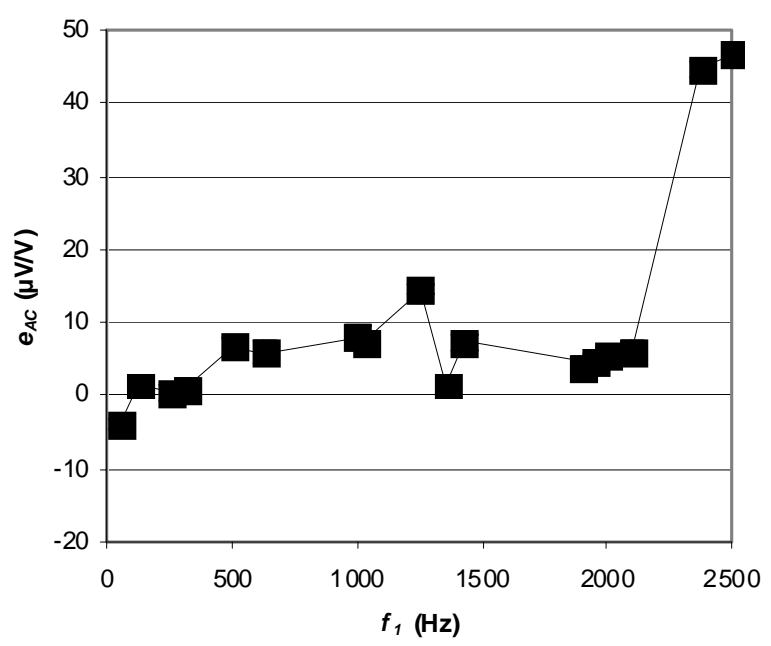

Fig.6. Relative error $e_{A C}$ of the DVM according to the frequency $f_{1}$ of the sine wave. The sampling parameters are $f_{e}=10.24 \mathrm{kHz}$ and $T_{a}=60 \mu \mathrm{s}$.

The highest harmonic frequency of the distorted waveform studied is $2500 \mathrm{~Hz}$. Then, first experiments have been performed to characterize the ADCs for frequencies from $400 \mathrm{~Hz}$ to $2500 \mathrm{~Hz}$. Fig. 6 shows the error $e_{A C}$ of the DVM (relative to the voltage reference value measured by AC-DC transfer technique) according to the frequency $f_{1}$ of the signal. The aperture time selected is $60 \mu \mathrm{s}$. The error varies from $-3 \mu \mathrm{V} / \mathrm{V}$ at $40 \mathrm{~Hz}$ to $+48 \mu \mathrm{V} / \mathrm{V}$ at $2500 \mathrm{~Hz}$. This error is taken into account in the estimation of the measurement uncertainty.

\section{B. Measurements of the RMS value of distorted waveforms}

For digitizing the signals, 1024 samples are taken over 5 periods and the sampling frequency is $10.24 \mathrm{kHz}$ to satisfy the Shannon condition. The aperture time is $60 \mu \mathrm{s}$. Twenty voltage waveforms are available, one sinusoidal waveform and nineteen distorted waveforms (table 1). The distorted waveforms have a dominant fundamental component at 50 $\mathrm{Hz}$ and one or several stationary harmonics. The THD varies from $0 \%$ (sine wave) to $84 \%$. Their amplitudes do not exceed $0.8 \mathrm{~V}$ and the DVM is used in the $1 \mathrm{~V}$ range.

Fig.7 represents the amplitude spectrum of a distorted signal containing a fundamental component at $50 \mathrm{~Hz}$ and two harmonics ( $k=3$ and $k=10)$. The uncertainty introduced by rounding the sample amplitudes to discrete levels can be viewed as adding quantization noise to the signal. The amount of this 'noise' decreases with increasing amplitude resolution. It can be expressed by the Signal-to-noise ratio (SNR) :

$$
(\mathrm{SNR})_{\mathrm{dB}} \approx 6 Q
$$

where $Q$ is the number of bits used to represent the signal. In our experimental configuration, 18 bits are used to represent the signal. It means that when measuring a $1 \mathrm{~V}$ input signal, the noise level is around $10^{-5} \mathrm{~V}$ according to the theory. As shown in Fig.7, experimental results are in good agreement with the theoretical calculation.

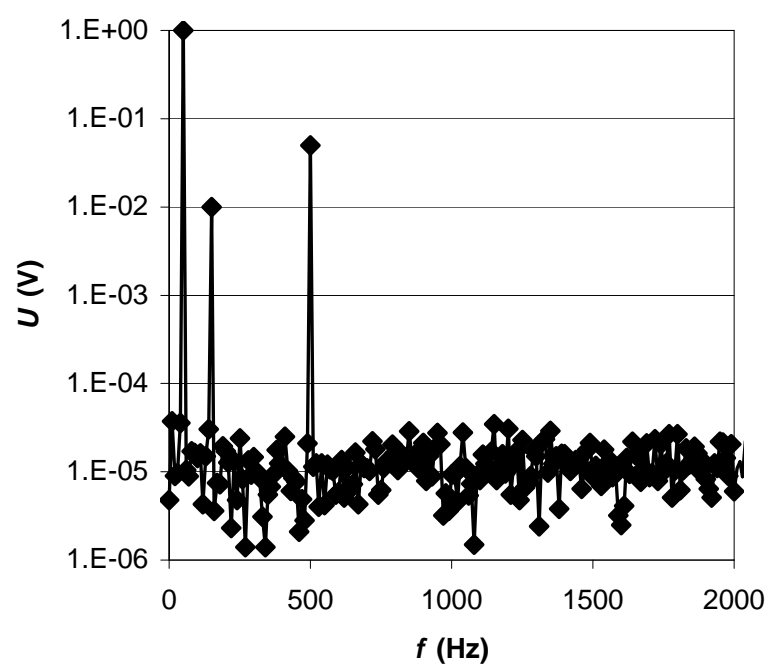

Fig.7. Example of amplitude spectrum for a distorted signal.

Fig.8 shows the error $e_{A C}$ of the DVM as a function of the THD of the distorted waveforms. It appears that the difference between the two techniques is always below 15 parts in $10^{6}$. Most of the measurements were repeatable to within 2 parts in $10^{6}$. 


\begin{tabular}{|c|c|c|}
\hline signal & Amplitude of the different harmonics & THD (\%) \\
\hline 1 & $U_{1}=1 \mathrm{~V}$ & 0 \\
\hline 2 & $U_{1}=1 \mathrm{~V}, U_{6}=0.01 \mathrm{~V}$ & 1 \\
\hline 3 & $U_{1}=1 \mathrm{~V}, U_{3}=0.01 \mathrm{~V}, U_{10}=0.05 \mathrm{~V}$ & 5.1 \\
\hline 4 & $\begin{array}{l}U_{1}=1 \mathrm{~V}, U_{5}=U_{15}=0.05 \mathrm{~V} \\
U_{30}=U_{40}=U_{50}=0.02 \mathrm{~V}\end{array}$ & 7.9 \\
\hline 5 & $U_{1}=1 \mathrm{~V}, U_{20}=0.1 \mathrm{~V}$ & 10 \\
\hline 6 & $U_{1}=1 \mathrm{~V}, U_{3}=U_{25}=U_{45}=0.1 \mathrm{~V}$ & 17.3 \\
\hline 7 & $\begin{array}{l}U_{1}=1 \mathrm{~V}, U_{2}=U_{3}=0.1 \mathrm{~V} \\
U_{4}=0.05 \mathrm{~V}, U_{5}=0.02 \mathrm{~V}, U_{50}=0.2 \mathrm{~V}\end{array}$ & 25.1 \\
\hline 8 & $U_{1}=1 \mathrm{~V}, U_{50}=0.3 \mathrm{~V}$ & 30 \\
\hline 9 & $\begin{array}{l}U_{1}=0.9 \mathrm{~V}, U_{2}=U_{36}=0.2 \mathrm{~V}, \\
U_{34}=U_{35}=0.1 \mathrm{~V}\end{array}$ & 35.1 \\
\hline 10 & $U_{1}=1 \mathrm{~V}, U_{3}=U_{4}=0.3 \mathrm{~V}$ & 42.4 \\
\hline 11 & $\begin{array}{l}U_{1}=0.8 \mathrm{~V}, U_{2}=0.25 \mathrm{~V}, U_{9}=0.25 \mathrm{~V} \\
U_{22}=U_{41}=U_{47}=0.1 \mathrm{~V}\end{array}$ & 49.2 \\
\hline 12 & $\begin{array}{l}U_{1}=0.8 \mathrm{~V}, U_{20}=0.3 \mathrm{~V}, \\
U_{36}=U_{50}=0.2 \mathrm{~V}\end{array}$ & 51.5 \\
\hline 13 & $\begin{array}{l}U_{1}=0.8 \mathrm{~V}, U_{48}=0.2 \mathrm{~V}, \\
U_{49}=U_{50}=0.3 \mathrm{~V}\end{array}$ & 58.6 \\
\hline 14 & $\begin{array}{l}U_{1}=0.8 \mathrm{~V}, U_{2}=U_{4}=U_{6}=U_{18}=U_{25}= \\
U_{26}=0.2 \mathrm{~V}, U_{27}=U_{28}=0.1 \mathrm{~V}\end{array}$ & 63.7 \\
\hline 15 & $\begin{array}{l}U_{1}=0.7 \mathrm{~V}, U_{2}=0.3 \mathrm{~V}, U_{13}=0.28 \mathrm{~V} \\
U_{34}=0.18 \mathrm{~V}\end{array}$ & 64 \\
\hline 16 & $\begin{array}{l}U_{1}=0.65 \mathrm{~V}, U_{22}=U_{34}=0.3 \mathrm{~V} \\
U_{40}=0.2 \mathrm{~V}\end{array}$ & 72.2 \\
\hline 17 & $\begin{array}{l}U_{1}=0.65 \mathrm{~V}, U_{4}=U_{5}=U_{6}=U_{7}=0.2 \mathrm{~V}, \\
U_{14}=U_{15}=U_{16}=U_{17}=U_{29}=U_{30}=0.1 \\
\mathrm{~V}\end{array}$ & 72.2 \\
\hline 18 & $\begin{array}{l}U_{1}=0,65 \mathrm{~V}, U_{22}=0,4 \mathrm{~V}, U_{34}=0,3 \mathrm{~V} \\
U_{40}=U_{50}=0,1 \mathrm{~V}\end{array}$ & 79.9 \\
\hline 19 & $\begin{array}{l}U_{1}=0.65 \mathrm{~V}, U_{2}=0.3 \mathrm{~V}, U_{3}=0.25 \mathrm{~V} \\
U_{4}=0.2 \mathrm{~V}, U_{5}=0.1 \mathrm{~V}, U_{6} \text { to } U_{32}=0.05 \\
\mathrm{~V}\end{array}$ & 79.9 \\
\hline 20 & $\begin{array}{l}U_{1}=0.65 \mathrm{~V}, U_{2}=U_{3}=0.3 \mathrm{~V} \\
U_{4}=U_{5}=U_{6}=0.2 \mathrm{~V}\end{array}$ & 84.3 \\
\hline
\end{tabular}

Table 1. Harmonic contents of the different signals measured.

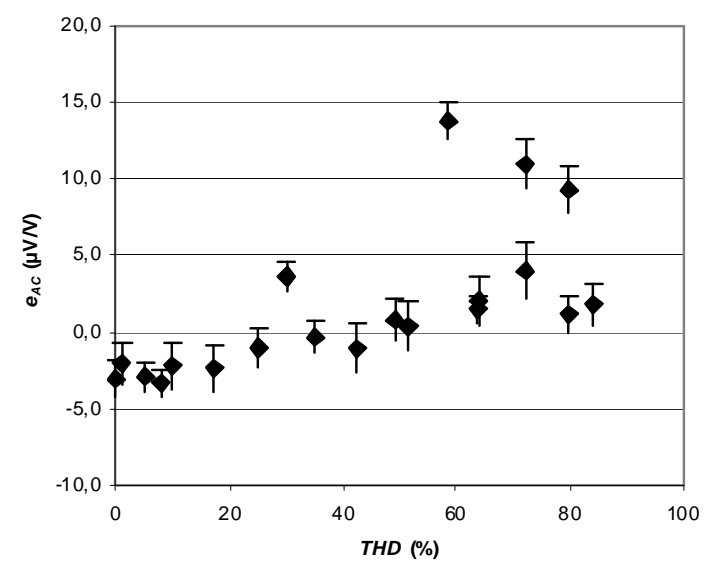

Fig.8. Relative error $e_{A C}$ of the DVM according to the THD of the distorted waveforms studied.

\section{Uncertainty analysis for the effective value.}

The uncertainty has been determined for the sampling of a spectrally "pure" (THD $<0.001 \%$ ) sinusoidal signal with the DVM operating in the "direct sampling” mode. Taking into account the different sources of errors [5], the relative uncertainty $(1 \sigma)$ is estimated to be $3 \mu \mathrm{V} / \mathrm{V}$ at $50 \mathrm{~Hz}$.

In our experiment, the signal has a dominant fundamental component at $50 \mathrm{~Hz}$ and its harmonic content is limited to
50 stationary harmonics (periodic signals). From (2), the relative uncertainty for the effective value $U_{\text {eff }}$ of this signal can be expressed as

$$
\left(\frac{\sigma_{U_{\text {eff }}}}{U_{\text {eff }}}\right)^{2}=\sum_{k=1}^{50}\left(\frac{U_{k}}{U_{\text {eff }}}\right)^{4} \cdot\left(\frac{\sigma_{U_{k}}}{U_{k}}\right)^{2}
$$

From the results presented in Fig.6, one can assume that the uncertainty of the term $\left(\frac{\sigma_{U_{k}}}{U_{k}}\right)$ is inferior to $50 \times 10^{-6}$ whatever the value of $k$. Besides, the ratio $\left(\frac{U_{k}}{U_{\text {eff }}}\right)$ does not exceed 50\% (tab.1).

Finally, the relative uncertainty for the effective value $U_{\text {eff }}$ is inferior to $25 \times 10^{-6}$.

\section{CONCLUSION}

A standard to measure RMS value of distorted voltage waveforms has been developed at LNE. Measurements have been performed on waveforms with THD ranging between 0 and $84 \%$. Good agreement with thermal converters, always better than 15 parts in $10^{6}$, has been obtained in all cases.

\section{ACKNOWLEDGMENT}

This research, conducted within the EURAMET joint research project 'Power and Energy', has received partial support from the European Community's Seventh Framework Programme, ERANET Plus, under Grant Agreement No. 217257.

* Identification of commercial equipment does not imply endorsement by LNE that it is the best available for the purpose.

\section{REFERENCES}

[1] Inglis, B.D. (1992). Standards for AC-DC transfer. Metrologia, 29 (2), 191-199.

[2] Klonz, M. (1997). CCE comparison of AC-DC voltage transfer standards at the lowest attainable level of uncertainty. IEEE Trans. Instrum. Meas., 46 (2), 342-346.

[3] Ilhenfeld, W.G.K. (2001). Maintenance and Traceability of AC Voltages by Synchronous Digital Synthesis and Sampling. Braunschweig, Germany: Physikalisch - Technische Bundesanstalt (PTB). (PTB Report E-75)

[4] Espel, P., Poletaeff, A., Bounouh, A. (2009). Static characterization of analog-to-digital converter. In Fundamental and Applied Metrology: XIX IMEKO World Congress, September 6-11, 2009, Lisbon, Portugal.

[5] Espel, P., Poletaeff, A., Bounouh, A. (2009). Characterization of analog-to-digital converters of a commercial digital voltmeter in the $20 \mathrm{~Hz}$ to $400 \mathrm{~Hz}$ frequency range. Metrologia, 46, 578-584. 